\title{
DESENVOLVIMENTO DE UM MODELO SIMPLIFICADO PARA A ATIVIDADE ELETROMECÂNICA CARDÍACA
}

\section{DEVELOPMENT OF A SIMPLIFIED MODEL FOR HEART ELECTROMECHANIC ACTIVITY}

\section{João Gabriel Rocha Silva ${ }^{\mathrm{a}, \mathrm{b}}$, Carolina Ribeiro Xavier ${ }^{\mathrm{c}}$, Ricardo Silva Campos $^{\mathrm{a}}$ e Rodrigo Weber dos Santos ${ }^{\mathrm{a}}$}

${ }^{a}$ Programa de Pós-Graduação em Modelagem Computacional, Universidade Federal de Juiz de Fora, Campus Universitário - Bairro Martelos, Juiz de Fora, Minas Gerais, Brasil, joaogabriel.comp@gmail.com,http://http://www.ufjf.br/pgmc/

${ }^{\mathrm{b}}$ Instituto Federal de Educação, Ciência e Tecnologia de Mato Grosso, Campus Pontes e Lacerda, Rodovia MT-473 s/n, Pontes e Lacerda, Mato Grosso, Brasil, joao.silva@ plc.edu.br, http://plc.ifmt.edu.br/inicio/

cPrograma de Pós-Graduação em Ciência da Computação, Universidade Federal de São João del Rei, Campus Tancredo Neves, São João del Rei, Minas Gerais, Brasil, carolrx@gmail.com, http://ppgcc.ufsj.edu.br/

Palavras-chave: Eletromecânica, modelagem computacional, modelos simplificados.

Resumo. Para o estudo do comportamento e medidas fisiológicas relacionados ao coração faz-se necessário uma análise e interpretação de sua atividade eletromecânica. Neste sentido, modelos matemáticocomputacionais são amplamente utilizados. Entretanto, estes modelos podem ser altamente custosos computacionalmente fazendo com que modelos simplificados, com um número menor de equações diferenciais, seja aplicado. Neste trabalho, é proposto um modelo matemático simplificado baseado em outros modelos simplificados da literatura. O modelo apresentado possui resultados qualitativos satisfatório inclusive quando simulado com diferentes períodos de potencial de ação.

Keywords:Electromechanical, computational modeling, simplified models.

\begin{abstract}
To study the behavior and physiological measurements related to the heart, it is necessary to analyze and interpret its electromechanical activity. In this sense, mathematical-computational models are widely used. However, these models can be highly computationally costly and simplified models with fewer differential equations are applied. This paper proposes a simplified mathematical model based on other simplified models in the literature. The model presented has satisfactory qualitative results even when simulated with different periods of action potential.
\end{abstract}




\section{INTRODUÇÃo}

O coração é um dos principais órgãos do corpo humano, caracterizado como um músculo que é capaz de bombear sangue para todo o corpo, permitindo assim, o carregamento de nutrientes por parte de cada célula (Berg et al., 2018).

Em uma questão anatômica o coração pode ser dividido em quatro grandes câmeras, dois átrios e dois ventrículos, os átrios possuem a função de receber o sangue do organismo enquanto os ventrículos possuem a função de expelir este sangue oxigenado para o corpo.

Um fator preocupante relacionado ao coração consistem em estatísticas envolvendo doenças relacionadas ao órgão. Estima-se que em 2013, doenças cardíacas em suas variações atingiram mais de 17,3 milhões de mortes no mundo. Esta taxa representa em torno de $31 \%$ de óbitos registrados no ano caracterizando a maior causa de mortes no ano estudado (Association et al., 2017).

Outro fator a se discutir é relacionado ao custo financeiro envolvendo pesquisas relacionados ao estudo, diagnóstico e tratamento relacionado à patologias cardíacas. Como por exemplo, em 2010, em torno de U\$ bilhões de dólares foram investidos neste sentido (Go et al., 2014).

Além do fato dos estudos envolvendo o coração serem de alto custo financeiro, estes procedimentos podem ser realizados de forma invasiva e desgastante para o paciente, como por exemplo, o implante de marca-passos (Izutani et al., 2002). Visto isto, modelos computacionais vem sendo aplicados visando a possibilidade de teste de drogas, equipamentos e geração de diagnósticos com um menor custo financeiro e um menor desgaste do paciente (Nataraj et al., 2012).

Modelos matemático-computacionais são modelos escrito através de equações matemáticas (diferenciais ordinárias, algébricas entre outras) com o intuito de a partir de uma implementação computacional para a matemática proposta simular um determinado fenômeno.

No âmbito do coração, modelos matemáticos são aplicados para o estudo de diferentes fenômenos, como por exemplo: geração de potencial de ação (Ten Tusscher et al., 2004) e estudo de propagação elétrica em redes de Purkinje (Ulysses et al., 2018).

Com o objetivo de desenvolver modelos computacionais para o estudo da atividade eletromecânica cardíaca (acoplamento entre o sistema elétrico e o sistema mecânico) diversos modelos foram propostos. Entretanto, grande parte deles devido a alta quantidade de aspectos físicoquímicos considerados, possuem alto custo computacional.

Este trabalho, apresenta um novo modelo para a atividade eletromecânica simplificado, isto é, com menos equações para representação do fenômeno, visando uma reprodução qualitativa da atividade mecânica apresentada por modelos complexos. Este modelo, é baseado em outros modelos simplificados da literatura.

\section{ELETROMECÂNICA CARDÍACA}

A atividade eletromecânica cardíaca pode ser descrita a partir de um potencial de ação, que consiste em um estímulo elétrico gerado por diferenças de concentrações iônicas entre o meio intra e extra-celular possibilitando um deslizamento dos filamentos de actina sobre os de miosina produzindo uma contração no músculo cardíaco. Nesta seção serão descritas as atividades elétrica (potencial de ação) e mecânica (contração celular).

\subsection{O potencial de Ação}

O potencial de ação consiste em um estímulo elétrico gerado por trocas de concentrações iônicas entre o meio intra e extra-celular, principalmente por íons de sódio e potássio. Este 
fenômeno é específico de células excitáveis, como: músculos e neurônios. A rápida propagação do potencial de ação por todo o coração é responsabilidade de células específicas que espalhem o efeito elétrico por todo o órgão.

Um período do potencial de ação é descrito em cinco fases: o repouso, que é o estado onde existe um equilíbrio entre os potenciais entre os meios intra e extra-celular. A despolarização, compreendida por um rápido crescimento na voltagem causado por um influxo de sódio. A repolarização inicial, que é uma pequena repolarização causada pelo efluxo de potássio e cálcio. O platô, que mantém uma estabilidade entre os meios intra e extra-celular sustentados por um influxo de cálcio. E a repolarização, onde ocorre uma forte queda na voltagem causada por um efluxo de sódio.

\subsection{Contração Celular}

Os sarcômeros são unidades fundamentais pra contração celular. Estes estão contidos nos miócitos cardíacos. Além disso, os sarcômeros são unidades que contém filamentos de actina e miosina, o deslizamento dos filamentos de actina sobre os filamentos de miosina provocam o encurtamento dos sarcômeros (contração das fibras musculares).

Esta contração é iniciada por um estímulo elétrico, este estímulo causa o aumento de cálcio no meio intracelular causando o deslizamento dos filamentos de actina sobre os de miosina causando a contração.

\section{MODELOS COMPUTACIONAIS PARA ELETROMECÂNICA CARDÍACA}

Modelos computacionais para o desenvolvimento da atividade eletromecânica cardíaca são geralmente baseados em dois modelos: um para a parte elétrica (geração de potencial de ação), outro para a parte mecânica (contração celular). Nesta sessão serão descritos modelos computacionais acoplados apresentados na literatura e estudos que basearam o presente trabalho.

\subsection{Lino (2010)}

Em (de Oliveira et al., 2010) foi proposto um modelo acoplado para a eletromecânica cardíaca baseado em dois modelos da literatura, para a parte elétrica (Ten Tusscher et al., 2004) com mais de 19 equações diferenciais. Para a parte mecânica o modelo utilizado foi o proposto por (Rice et al., 2008) apoiado por 11 equações diferenciais e mais de 45 equações algébricas.

Para o acoplamento entre os modelos foi necessário um ajuste de parâmetros feito por um algoritmo genético. A Figura 1 apresenta o potencial de ação e a tensão ativa (contração) desenvolvido por (de Oliveira et al., 2010). O resultado foi descrito como satisfatório pelos autores baseado no fato de dados experimentais de humanos estarem com valores próximos à esta simulação.

\subsection{Iribe (2006)}

Outro modelo computacional acoplado foi apresentado em (Iribe et al., 2006). Este modelo utiliza-se do modelo para geração de potencial elétrico proposto por (Noble et al., 1991), tratase de um modelo complexo, com muitas considerações e inúmeras equações diferenciais para apoio à representação.

Quanto à parte mecânica, o modelo de (Rice et al., 1999) foi utilizado. Este, baseado em 7 equações diferenciais e 19 equações algébricas.

Para o acoplamento dos modelos a proposta utilizada baseia-se na concentração de cálcio intracelular, outras considerações podem ser verificadas em (Iribe et al., 2006). A Figura 2 


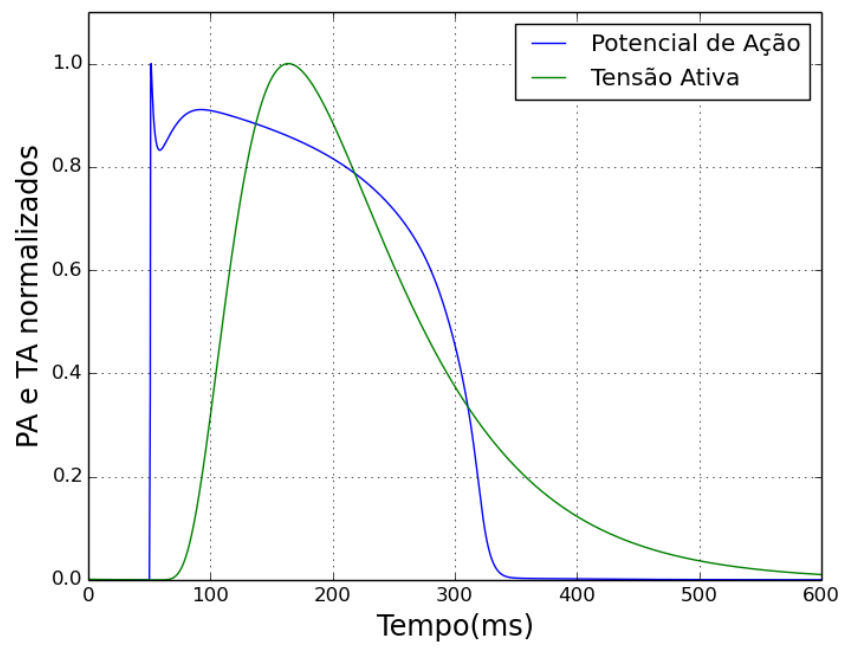

Figura 1: Potencial de Ação e Tensão Ativa desenvolvido em (de Oliveira et al., 2010)

apresenta o resultado do acoplamento proposto para esta combinação de modelos para diferentes períodos de potencial de ação no estado estacionário, respectivamente: (a) $0.4 \mathrm{~ms}$, (b) $0.5 \mathrm{~ms}$ e (c) $0.75 \mathrm{~ms}$ de duração.

\subsection{Silva (2017)}

Em (Silva et al., 2017) são propostos dois modelos simplificados para o acoplamento eletromecânico cardíaco, estes modelos foram baseados no modelo celular de (Ten Tusscher et al., 2004) acoplado a modelos para tensão ativa simplificados da literatura, gerando o Modelo A, baseado em (Nash e Panfilov, 2004) e Modelo B, baseado em (Göktepe e Kuhl, 2010).

Estes modelos foram criados a partir de quatro etapas complementares, e são apoiados por apenas 2 equações diferenciais ordinários e 3 equações algébricas para a parte mecânica. No acoplamento, um algoritmo genético foi utilizado com o intuito de aproximar a curva de tensão ativa destes modelos simplificados do modelo detalhado de (de Oliveira et al., 2010).

A Figura 3 apresenta o potencial de ação e a tensão ativa dos modelos propostos junto ao modelo utilizado como referência ((de Oliveira et al., 2010)). Na Figura é possível verificar que qualitativamente os resultados para estas simulações são satisfatórios mesmo diminuindo cerca de 9 equações diferenciais e mais de 40 equações algébricas.

\section{MODELO PROPOSTO}

Baseado no estudo dos modelos acoplados mencionados, propusemos uma modificação nos modelos A e B de (Silva et al., 2017) trabalhando em uma equação algébrica em cada um dos modelos (a única equação que difere o Modelo A do B) baseado no fato desta equação após o ajuste via algoritmo genético ter o comportamento similar ao de uma constante. A Equação 1 no Modelo A e a Equação 2 no Modelo B serão substituídas por uma constante $\left(c_{0}\right)$ gerando um único Modelo, que é descrito pelas Equações 3 com destaque nos parâmetros em vermelho que serão submetidos a ajustes via algoritmo genético.

Nesta proposta, o modelo utilizado para a geração de potencial de ação (elétrica) permanece 


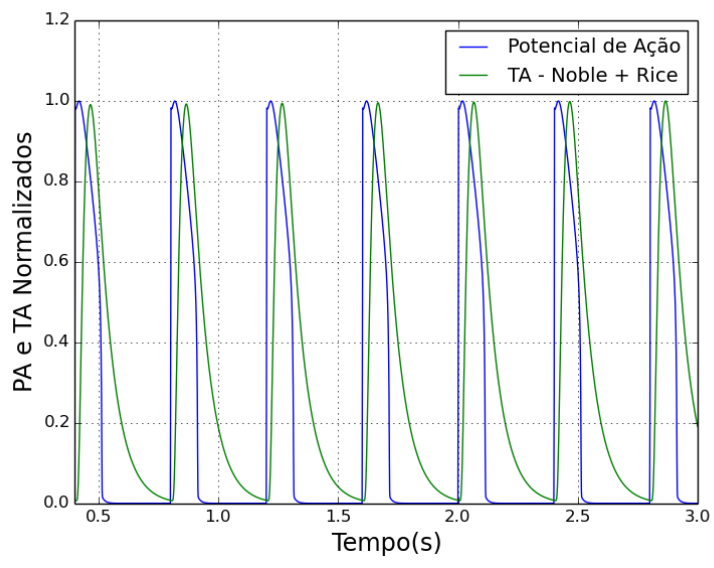

(a) $0.4 \mathrm{~ms}$

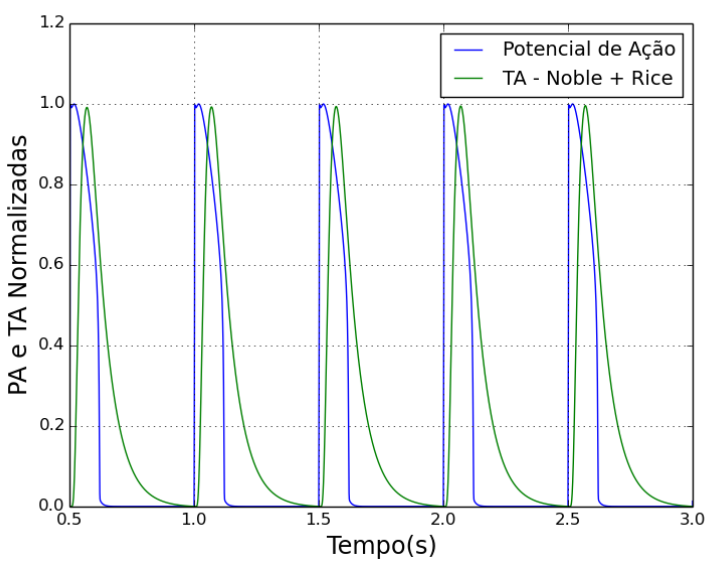

(b) $0.5 \mathrm{~ms}$

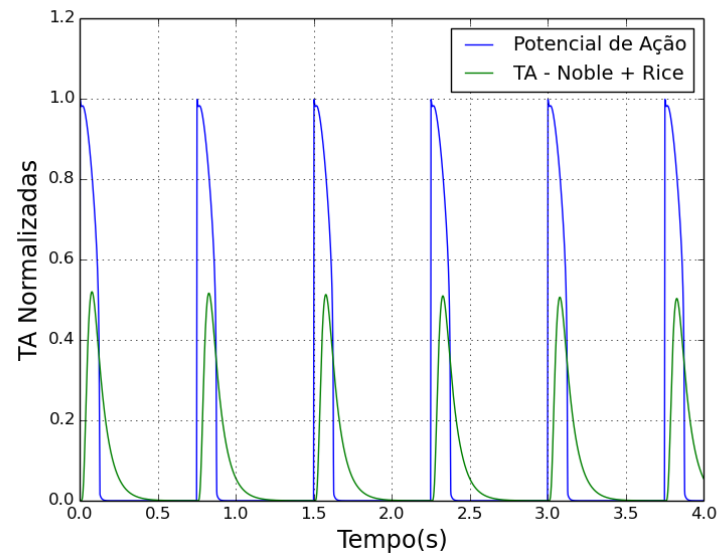

(c) $0.75 \mathrm{~ms}$

Figura 2: Potencial de ação e tensão ativa proposto por (Iribe et al., 2006) para diferentes períodos

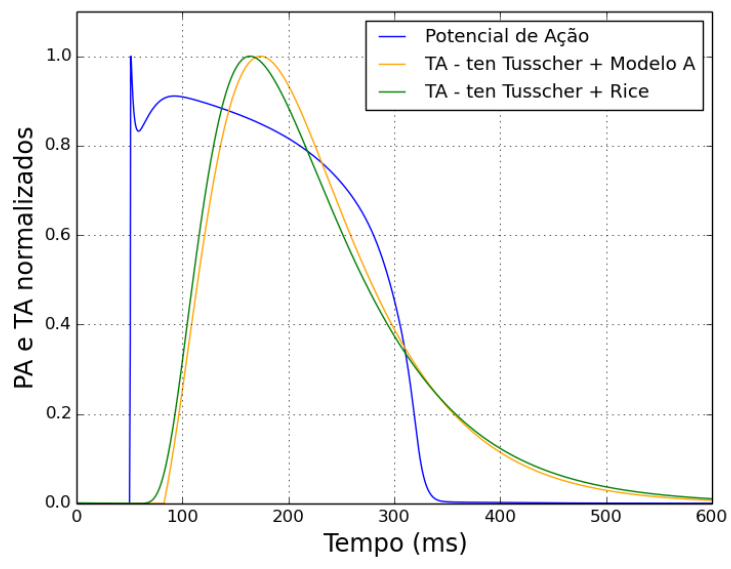

(a) Modelo A

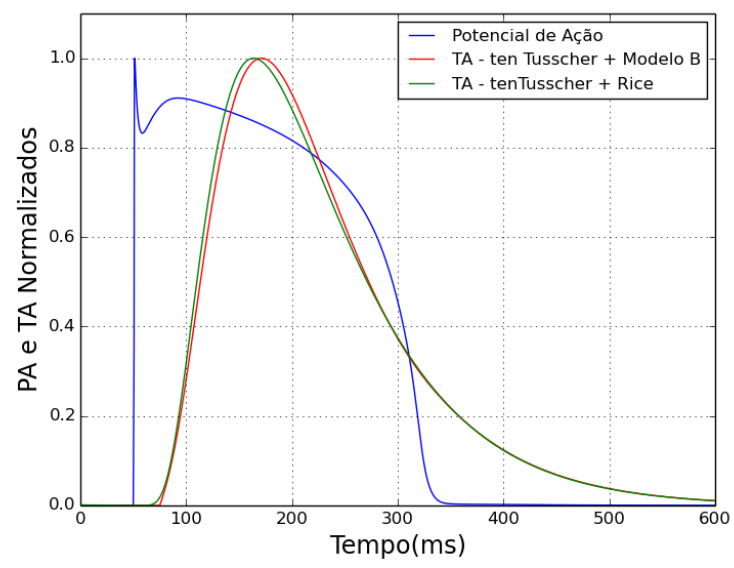

(b) Modelo B

Figura 3: Potencial de ação e tensão ativa dos modelos apresentados junto ao modelo referência 
o utilizado por (Silva et al., 2017), o modelo celular de (Ten Tusscher et al., 2004).

$$
\begin{array}{r}
\epsilon_{0}(V)=\left\{\begin{array}{cc}
e_{0} & \text { para } \mathrm{V}<e_{t} \\
10 e_{0} & \text { para } \mathrm{V} \geq e_{t}
\end{array}\right. \\
\epsilon_{0}(V)=\left(e_{0}+\left(e_{\infty}-e_{0}\right) e^{-e^{(-\xi(V-v s))}}\right) \\
\frac{\mathrm{d} T a_{i}}{\mathrm{~d} t}=c_{0}\left(k(V)-T a_{i}\right) \\
\frac{\mathrm{d} T a}{\mathrm{~d} t}=\epsilon_{1}\left(V, T a_{i}\right)\left(T a_{i}-T a\right) \\
\epsilon_{1}(V)= \begin{cases}x_{1} & \text { para } \mathrm{V}>x_{2} \mathrm{e} T a_{i}<x_{3} \\
c_{0} & \text { caso contrário }\end{cases} \\
k(V)=\frac{1}{\sigma \sqrt{2 \pi}} e^{\frac{-1}{2}\left(\frac{V-1}{\sigma}\right)^{2}}
\end{array}
$$

\section{RESULTADOS ALCANÇADOS}

Dois experimentos foram realizados para a validação do novo modelo proposto. Nesta sessão segue a descrição e resultados de cada um deles.

\subsection{Experimento 1}

O primeiro experimento consiste na submissão dos parâmetros do modelo a ajuste via algoritmo genético com o intuito de reproduzir os resultados alcançados pelos Modelos A e B de (Silva et al., 2017). Onde busca-se a minimização da diferença entre a tensão ativa para este modelo simplificado quando comparado ao modelo complexo desenvolvido em (de Oliveira et al., 2010).

A Figura 4 apresenta a tensão ativa para o modelo eletromecânico proposto a partir do ajuste de parâmetros realizados via algoritmo genético. Nota-se que o novo modelo consegue um desempenho satisfatório quando comparado ao modelo complexo de (de Oliveira et al., 2010) e também quando comparado aos modelos de (Silva et al., 2017) onde para estes, uma equação algébrica é diminuída.

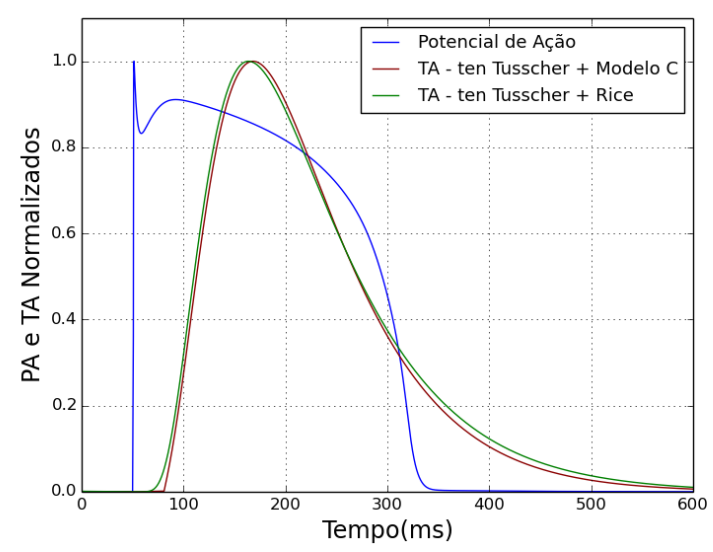

Figura 4: Potencial de Ação e Tensão Ativa para o modelo eletromecânico C após ajuste via algoritmo genético 
Na Tabela 1 são apresentados os parâmetros finais obtidos pelo ajuste de parâmetros pelo AG para o modelo acoplado C.

\begin{tabular}{|c|c|}
\hline Parâmetro & Valor \\
\hline$c_{0}$ & 0.016602 \\
\hline$\sigma$ & 0.042969 \\
\hline$x_{1}$ & 0.0001 \\
\hline$x_{2}$ & $0.827044 \mathrm{mV}$ \\
\hline$x_{3}$ & 0.209961 \\
\hline
\end{tabular}

Tabela 1: Parâmetros finais obtidos pelo AG no Modelo C.

\subsection{Experimento 2}

O segundo experimento consiste na reprodução da tensão ativa gerada pelo modelo acoplado de (Iribe et al., 2006) utilizando o Modelo proposto neste trabalho para diferentes períodos de potencial de ação utilizando o algoritmo genético para ajuste de parâmetros.

A Figura 5 apresenta as tensões ativas (contração) geradas pelo modelo $\mathrm{C}$ para diferentes períodos de duração do potencial de ação no estado estacionário para (a) $0.4 \mathrm{~ms}$, (b) $0.5 \mathrm{~ms}$ e (c) $0.75 \mathrm{~ms}$ comparado à tensão ativa para os mesmos períodos propostos pelo modelo de (Iribe et al., 2006) (Noble+Rice).

É possível observar na Figura 5 que o modelo proposto reproduz de forma satisfatória qualitativamente a tensão ativa do modelo apresentado por (Iribe et al., 2006) com a vantagem de contar com 5 equações diferencias e 17 equações algébricas a menos.

A Tabela 2 apresenta os parâmetros encontrados para o ajuste desta reprodução no estado estacionário para o período de $0.4 \mathrm{~s}$.

\begin{tabular}{|c|c|}
\hline Parâmetro & Valor \\
\hline$c_{0}$ & 0.023438 \\
\hline$\sigma$ & 0.053711 \\
\hline$x_{1}$ & 0.008789 \\
\hline$x_{2}$ & $0.646485 \mathrm{mV}$ \\
\hline$x_{3}$ & 0.655274 \\
\hline
\end{tabular}

Tabela 2: Parâmetros obtidos pelo AG para a reprodução do estado estacionário com estímulos de frequência 0.4.

A Tabela 3 apresenta os parâmetros encontrados para o ajuste da reprodução da tensão ativa a partir de um período de $0.5 \mathrm{~ms}$ no estado estacionário.

\begin{tabular}{|c|c|}
\hline Parâmetro & Valor \\
\hline$c_{0}$ & 0.022461 \\
\hline$\sigma$ & 0.037109 \\
\hline$x_{1}$ & 0.003906 \\
\hline$x_{2}$ & $0.952149 \mathrm{mV}$ \\
\hline$x_{3}$ & 0.921875 \\
\hline
\end{tabular}

Tabela 3: Parâmetros obtidos pelo AG para a reprodução do estado estacionário com estímulos de período $0.5 \mathrm{~s}$. 


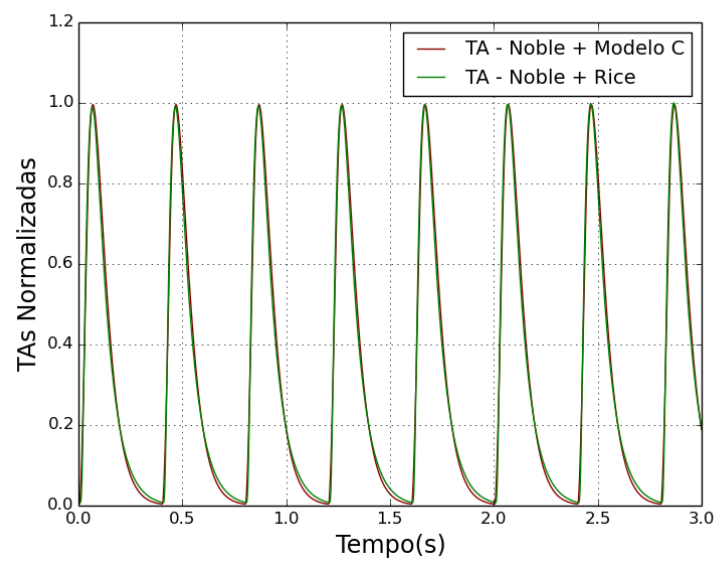

(a) $0.4 \mathrm{~ms}$

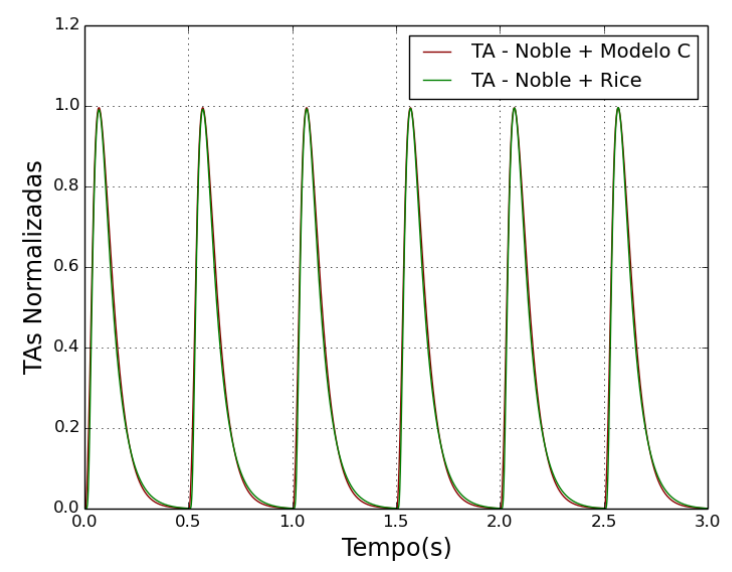

(b) $0.5 \mathrm{~ms}$

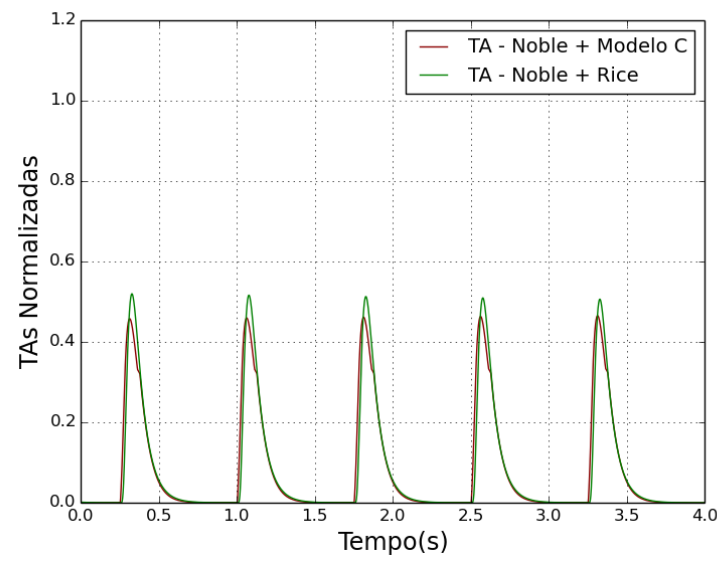

(c) $0.75 \mathrm{~ms}$

Figura 5: Tensões ativas para o modelo C e o Modelo proposto por (Iribe et al., 2006)

A Tabela 4 apresenta os parâmetros encontrados para o ajuste da reprodução da tensão ativa no estado estacionário para o período de $0.75 \mathrm{~s}$.

\section{CONCLUSÕES}

Neste trabalho propusemos um novo modelo simplificado para a atividade eletromecânica cardíaca. Este, com um menor custo computacional quando comparados a outros modelos da literatura, visto basear-se em menos equações para representação.

Nosso modelo possui bons resultados quando submetido à comparações qualitativas diretas com outros modelos computacionais simplificados, bem como quando comparados a diferentes períodos de potencial de ação no estado estacionário.

Trabalhos futuros a serem desenvolvidos consistem na implementação de novas heurísticas para ajuste de parâmetros do modelo, como por exemplo: grasp e evolução diferencial. Além disso, propor um modelo simplificado específico para geração de potencial de ação, gerando assim um modelo com ainda menos equações diferenciais. 


\begin{tabular}{|c|c|}
\hline Parâmetro & Valor \\
\hline$c_{0}$ & 0.020508 \\
\hline$\sigma$ & 0.013672 \\
\hline$x_{1}$ & 0.003906 \\
\hline$x_{2}$ & $0.017578 \mathrm{mV}$ \\
\hline$x_{3}$ & 0.996094 \\
\hline
\end{tabular}

Tabela 4: Parâmetros obtidos pelo AG para a reprodução do estado estacionário com estímulos de frequência $0.75 \mathrm{~s}$.

\section{AGRADECIMENTOS:}

Os autores agradecem à Coordenação de Aperfeiçoamento de Pessoal de Nível Superior (Capes), Universidade Federal de Juiz de Fora (UFJF), Universidade Federal de São João del Rei (UFSJ) e Instituto Federal de Educação, Ciência e Tecnologia de Mato Grosso (IFMT).

\section{REFERÊNCIAS}

Association A.H. et al. Heart disease and stroke statistics 2017 at-a-glance. Geraadpleegd van: https://healthmetrics. heart. org/wp-content/uploads/2017/06/Heart-Disease-andStroke-Statistics-2017-ucm_491265. pdf, 2017.

Berg L.A. et al. Estudo da propagação elétrica em fibras de purkinje. 2018.

de Oliveira B.L., Sundnes J., e dos Santos R.W. The development of a new computational model for the electromechanics of the human ventricular myocyte. Em Engineering in Medicine and Biology Society (EMBC), 2010 Annual International Conference of the IEEE, páginas 38203823. IEEE, 2010.

Go A.S., Mozaffarian D., Roger V.L., Benjamin E.J., Berry J.D., Blaha M.J., Dai S., Ford E.S., Fox C.S., et al. Heart disease and stroke statistics-2014 update: a report from the american heart association. circulation, 129(3):e28, 2014.

Göktepe S. e Kuhl E. Electromechanics of the heart: a unified approach to the strongly coupled excitation-contraction problem. Computational Mechanics, 45(2):227-243, 2010.

Iribe G., Kohl P., e Noble D. Modulatory effect of calmodulin-dependent kinase ii (camkii) on sarcoplasmic reticulum ca2+ handling and interval-force relations: a modelling study. Philosophical Transactions of the Royal Society of London A: Mathematical, Physical and Engineering Sciences, 364(1842):1107-1133, 2006.

Izutani H., Quan K.J., Biblo L.A., e Gill I.S. Biventricular pacing for congestive heart failure: early experience in surgical epicardial versus coronary sinus lead placement. The heart surgery forum, 6(1):E1-6; discussion E1-6, 2002. ISSN 1522-6662. PMID: 12611737.

Nash M.P. e Panfilov A.V. Electromechanical model of excitable tissue to study reentrant cardiac arrhythmias. Progress in biophysics and molecular biology, 85(2):501-522, 2004.

Nataraj C., Jalali A., e Ghorbanian P. Application of computational intelligence techniques for cardiovascular diagnostics. Em The Cardiovascular System-Physiology, Diagnostics and Clinical Implications. InTech, 2012.

Noble D., Noble S., Bett G., Earm Y., Ho W., e So I. The role of sodium-calcium exchange during the cardiac action potential. Annals of the New York Academy of Sciences, 639(1):334353, 1991.

Rice J.J., Wang F., Bers D.M., e De Tombe P.P. Approximate model of cooperative activation and crossbridge cycling in cardiac muscle using ordinary differential equations. Biophysical journal, 95(5):2368-2390, 2008.

Rice J.J., Winslow R.L., e Hunter W.C. Comparison of putative cooperative mechanisms in 
cardiac muscle: length dependence and dynamic responses. American Journal of PhysiologyHeart and Circulatory Physiology, 276(5):H1734-H1754, 1999.

Silva J.G.R., Rocha B.M., Campos R.S., Xavier C.R., e dos Santos R.W. Modelos simplificados para acoplamento eletromecânico de miócito cardíaco. Em XXIII Congreso sobre Métodos Numéricos y sus Aplicaciones. 2017.

Ten Tusscher K., Noble D., Noble P., e Panfilov A.V. A model for human ventricular tissue. American Journal of Physiology-Heart and Circulatory Physiology, 286(4):H1573-H1589, 2004.

Ulysses J.N., Berg L.A., Cherry E.M., Liu B.R., dos Santos R.W., de Barros B.G., Rocha B.M., e de Queiroz R.A. An optimization-based algorithm for the construction of cardiac purkinje network models. IEEE Transactions on Biomedical Engineering, 65(12):2760-2768, 2018. 